Pacific

Journal of

Mathematics

\title{
ON THE EQUIVALENCE OF THE DEFINITIONS OF VOLUME OF REPRESENTATIONS
}

SUNGWOON KIM 


\title{
ON THE EQUIVALENCE OF THE DEFINITIONS OF VOLUME OF REPRESENTATIONS
}

\author{
SUNGWOON KIM
}

Let $G$ be a rank-1 simple Lie group and let $M$ be a connected, orientable, aspherical, tame manifold. Assume that each end of $M$ has amenable fundamental group. There are several definitions of volume of representations of $\pi_{1}(M)$ into $G$. We give a new definition of volume of representations and furthermore show that all definitions so far are equivalent.

\section{Introduction}

Let $G$ be a semisimple Lie group and let $\mathcal{X}$ be the associated symmetric space of dimension $n$. Let $M$ be a connected, orientable, aspherical, tame manifold of the same dimension as $\mathcal{X}$. First assume that $M$ is compact. To each representation $\rho: \pi_{1}(M) \rightarrow G$, one can associate a volume of $\rho$ in the following way. First, associate a flat bundle $E_{\rho}$ over $M$ with fiber $\mathcal{X}$ to $\rho$. Since $\mathcal{X}$ is contractible, there always exists a section $s: M \rightarrow E_{\rho}$. Let $\omega_{\mathcal{X}}$ be the Riemannian volume form on $\mathcal{X}$. One may think of $\omega_{\mathcal{X}}$ as a closed differential form on $E_{\rho}$ by spreading $\omega_{\mathcal{X}}$ over the fibers of $E_{\rho}$. Then the volume of $\rho$ is defined by

$$
\operatorname{Vol}(\rho)=\int_{M} s^{*} \omega_{\mathcal{X}}
$$

Since any two sections are homotopic to each other, the volume $\operatorname{Vol}(\rho)$ does not depend on the choice of section.

The volume of representations has been used to characterize discrete faithful representations. Let $\Gamma$ be a uniform lattice in $G$. Then the volume of representations satisfies a Milnor-Wood type inequality. More precisely, for any representation $\rho: \Gamma \rightarrow G$, we have

$$
|\operatorname{Vol}(\rho)| \leq \operatorname{Vol}(\Gamma \backslash \mathcal{X})
$$

This research was supported by the Basic Science Research Program through the National Research Foundation of Korea (NRF) funded by the Ministry of Education, Science and Technology (NRF-2012R1A1A2040663).

MSC2010: 53C24, 53C 35 .

Keywords: Lie groups, representation, volume, symmetric spaces, disjoint cone. 
Furthermore, equality holds in (1) if and only if $\rho$ is discrete and faithful. This is the so-called volume rigidity theorem. Goldman [1982] proved the volume rigidity theorem in the higher rank case and Besson, Courtois and Gallot [Besson et al. 2007] proved the theorem in the rank-1 case.

Now assume that $M$ is noncompact. Then the definition of volume of representations as above is not valid anymore since some problems of integrability arise. So far, three definitions of volume of representations have been given under some conditions on $M$. Let us first fix the following notation throughout the paper.

Setup. Let $M$ be a noncompact, connected, orientable, aspherical, tame manifold. Denote by $\bar{M}$ the compact manifold with boundary whose interior is homeomorphic to $M$. Assume that each connected component of $\partial \bar{M}$ has amenable fundamental group. Let $G$ be a rank-1 semisimple Lie group with trivial center and no compact factors. Let $\mathcal{X}$ be the associated symmetric space of dimension $n$. Assume that $M$ has the same dimension as $\mathcal{X}$.

First of all, Dunfield [1999] introduced the notion of pseudodeveloping map to define the volume of representations of a nonuniform lattice $\Gamma$ in $\operatorname{SO}(3,1)$. It was successful in making an invariant associated with a representation $\rho: \Gamma \rightarrow \operatorname{SO}(3,1)$ but he did not prove that the volume of representations does not depend on the chosen pseudodeveloping map. After that, Francaviglia [2004] proved the well-definedness of the volume of representations. Then Francaviglia and Klaff [2006] extended the definition of volume of representations and the volume rigidity theorem to general nonuniform hyperbolic lattices. We call the definition of volume of representations via pseudodeveloping map D1. For more detail about D1, see [Francaviglia and Klaff 2006] or Section 4.

The second definition D2 of volume of representations was given by Bucher, Burger and Iozzi [Bucher et al. 2013] and generalizes the one introduced in [Burger et al. 2010] for noncompact surfaces. They used the theory of bounded cohomology to make an invariant associated with a representation. Given a representation $\rho: \pi_{1}(M) \rightarrow G$, one cannot get any information from the pullback map in degree $n$ in continuous cohomology, $\rho_{c}^{*}: H_{c}^{n}(G, \mathbb{R}) \rightarrow H^{n}\left(\pi_{1}(M), \mathbb{R}\right)$, since $H^{n}\left(\pi_{1}(M), \mathbb{R}\right) \cong$ $H^{n}(M, \mathbb{R})$ is trivial. However, the situation is different in continuous bounded cohomology. Not only may the pullback map $\rho_{b}^{*}: H_{c, b}^{n}(G, \mathbb{R}) \rightarrow H_{b}^{n}\left(\pi_{1}(M), \mathbb{R}\right)$ be nontrivial but it also encodes subtle algebraic and topological properties of a representation such as injectivity and discreteness. Bucher, Burger and Iozzi gave a proof of the volume rigidity theorem for representations of hyperbolic lattices from the point of view of bounded cohomology. We refer the reader to [Bucher et al. 2013] or Section 2 for further discussion about D2.

Recently, S. Kim and I. Kim [2014] gave a new definition, called D3, of volume of representations in the case that $M$ is a complete Riemannian manifold with 
finite Lipschitz simplicial volume. See [Kim and Kim 2014] or Section 5 for the exact definition of D3. In D3, it is not necessary that each connected component of $\partial \bar{M}$ has amenable fundamental group, while the amenable condition on $\partial \bar{M}$ is necessary in D2. They only use the bounded cohomology and $\ell^{1}$-homology of $M$. It is quite useful to define the volume of representations in the case that the amenable condition on $\partial \bar{M}$ does not hold. They give a proof of the volume rigidity theorem for representations of lattices in an arbitrary semisimple Lie group in their setting.

In this note, we will give another definition of volume of representations, called D4. In D4, $\rho$-equivariant maps are involved as in D1 and the bounded cohomology of $M$ is involved as in D2 and D3. In fact, D4 seems to be a kind of definition connecting the other definitions D1, D2 and D3. Eventually we show that all the definitions are equivalent.

Theorem 1.1. Let $G$ be a rank-1 simple Lie group with trivial center and no compact factors. Let $M$ be a noncompact, connected, orientable, aspherical, tame manifold. Suppose that each end of $M$ has amenable fundamental group. Then all definitions D1,D2 and D3 of volume of representations of $\pi_{1}(M)$ into $G$ are equivalent. Furthermore, if $M$ admits a complete Riemannian metric with finite Lipschitz simplicial volume, all definitions D1,D2,D3 and D4 are equivalent.

The paper is organized as follows. For our proof, we recall the definitions of volume of representations in the order D2, D4, D1, D3. In Section 2, we first recall definition D2. In Section 3, we give definition D4 and then prove that D2 and D4 are equivalent. In Section 4, after recalling definition D1, we show the equivalence of D1 and D4. Finally in Section 5, we complete the proof of Theorem 1.1 by proving that D3 and D4 are equivalent.

\section{Bounded cohomology and definition D2}

We choose the appropriate complexes for the continuous cohomology and continuous bounded cohomology of $G$ for our purpose. Consider the complex $C_{c}^{*}(\mathcal{X}, \mathbb{R})_{\text {alt }}$ with the homogeneous coboundary operator, where

$$
C_{c}^{k}(\mathcal{X}, \mathbb{R})_{\mathrm{alt}}=\left\{f: \mathcal{X}^{k+1} \rightarrow \mathbb{R} \mid f \text { is continuous and alternating }\right\} .
$$

The action of $G$ on $C_{c}^{k}(\mathcal{X}, \mathbb{R})$ alt is given by

$$
g \cdot f\left(x_{0}, \ldots, x_{k}\right)=f\left(g^{-1} x_{0}, \ldots, g^{-1} x_{k}\right) .
$$

Then the continuous cohomology $H_{c}^{*}(G, \mathbb{R})$ can be isomorphically computed by the cohomology of the $G$-invariant complex $C_{c}^{*}(\mathcal{X}, \mathbb{R}){ }_{\text {alt }}^{G}$ (see [Guichardet 1980, Chapitre III]). According to the Van Est isomorphism [Borel and Wallach 2000, Proposition IX.5.5], the continuous cohomology $H_{c}^{*}(G, \mathbb{R})$ is isomorphic to the set 
of $G$-invariant differential forms on $\mathcal{X}$. Hence, in degree $n, H_{c}^{n}(G, \mathbb{R})$ is generated by the Riemannian volume form $\omega_{\mathcal{X}}$ on $\mathcal{X}$.

Let $C_{c, b}^{k}(\mathcal{X}, \mathbb{R})_{\text {alt }}$ be a subcomplex of continuous, alternating, bounded realvalued functions on $\mathcal{X}^{k+1}$. The continuous bounded cohomology $H_{c, b}^{*}(G, \mathbb{R})$ is obtained by the cohomology of the $G$-invariant complex $C_{c, b}^{*}(\mathcal{X}, \mathbb{R})_{\text {alt }}^{G}$ (see [Monod 2001, Corollary 7.4.10]). The inclusion of complexes $C_{c, b}^{*}(\mathcal{X}, \mathbb{R})_{\text {alt }}^{G} \subset C_{c}^{*}(\mathcal{X}, \mathbb{R})_{\text {alt }}^{G}$ induces a comparison map $H_{c, b}^{*}(G, \mathbb{R}) \rightarrow H_{c}^{*}(G, \mathbb{R})$.

Let $Y$ be a countable $C W$-complex. Denote by $C_{b}^{k}(Y, \mathbb{R})$ the complex of bounded real-valued $k$-cochains on $Y$. For a subspace $B \subset Y$, let $C_{b}^{k}(Y, B, \mathbb{R})$ be the subcomplex of those bounded $k$-cochains on $Y$ that vanish on simplices with image contained in $B$. The complexes $C_{b}^{*}(Y, \mathbb{R})$ and $C_{b}^{*}(Y, B, \mathbb{R})$ define the bounded cohomologies $H_{b}^{*}(Y, \mathbb{R})$ and $H_{b}^{*}(Y, B, \mathbb{R})$ respectively. For our convenience, we give another complex which computes the bounded cohomology $H_{b}^{*}(Y, \mathbb{R})$ of $Y$. Let $C_{b}^{k}(\tilde{Y}, \mathbb{R})_{\text {alt }}$ denote the complex of bounded, alternating real-valued Borel functions on $(\tilde{Y})^{k+1}$. The $\pi_{1}(Y)$-action on $C_{b}^{*}(\widetilde{Y}, \mathbb{R})_{\text {alt }}$ is defined as the $G$-action on $C_{c}^{*}(\mathcal{X}, \mathbb{R})$. Ivanov [1985] proved that the $\pi_{1}(Y)$-invariant complex $C_{b}^{*}(\widetilde{Y}, \mathbb{R})_{\text {alt }}^{\pi_{1}(Y)}$ defines the bounded cohomology of $Y$.

Bucher, Burger and Iozzi [Bucher et al. 2013] used bounded cohomology to define the volume of representations. Let $\bar{M}$ be a connected, orientable, compact manifold with boundary. Suppose that each component of $\partial \bar{M}$ has amenable fundamental group. In that case, it is proved in [Bucher et al. 2012; Kim and Kuessner 2015] that the natural inclusion $i:(\bar{M}, \varnothing) \rightarrow(\bar{M}, \partial \bar{M})$ induces an isometric isomorphism in bounded cohomology,

$$
i_{b}^{*}: H_{b}^{*}(\bar{M}, \partial \bar{M}, \mathbb{R}) \rightarrow H_{b}^{*}(\bar{M}, \mathbb{R}),
$$

in degrees $* \geq 2$. Noting the remarkable result of Gromov [1982, Section 3.1] that the natural map $H_{b}^{n}\left(\pi_{1}(\bar{M}), \mathbb{R}\right) \rightarrow H_{b}^{n}(\bar{M}, \mathbb{R})$ is an isometric isomorphism in bounded cohomology, for a given representation $\rho: \pi_{1}(M) \rightarrow G$ we have a map

$$
\rho_{b}^{*}: H_{c, b}^{n}(G, \mathbb{R}) \rightarrow H_{b}^{n}\left(\pi_{1}(\bar{M}), \mathbb{R}\right) \cong H_{b}^{n}(\bar{M}, \mathbb{R}) \cong H_{b}^{n}(\bar{M}, \partial \bar{M}, \mathbb{R}) .
$$

The $G$-invariant Riemannian volume form $\omega_{\mathcal{X}}$ on $\mathcal{X}$ gives rise to a continuous bounded cocycle $\Theta: \mathcal{X}^{n+1} \rightarrow \mathbb{R}$ defined by

$$
\Theta\left(x_{0}, \ldots, x_{n}\right)=\int_{\left[x_{0}, \ldots, x_{n}\right]} \omega_{\mathcal{X}},
$$

where $\left[x_{0}, \ldots, x_{n}\right]$ is the geodesic simplex with ordered vertices $x_{0}, \ldots, x_{n}$ in $\mathcal{X}$. The boundedness of $\Theta$ is due to the fact that the volume of geodesic simplices in $\mathcal{X}$ is uniformly bounded from above [Inoue and Yano 1982]. Hence the cocycle $\Theta$ induces a continuous cohomology class $[\Theta]_{c} \in H_{c}^{n}(G, \mathbb{R})$ and, moreover, a continuous bounded cohomology class $[\Theta]_{c, b} \in H_{c, b}^{n}(G, \mathbb{R})$. The image of $\left(\left(i_{b}^{*}\right)^{-1} \circ \rho_{b}^{*}\right)[\Theta]_{c, b}$ 
via the comparison map $c: H_{b}^{n}(\bar{M}, \partial \bar{M}, \mathbb{R}) \rightarrow H^{n}(\bar{M}, \partial \bar{M}, \mathbb{R})$ is an ordinary relative cohomology class. Its evaluation on the relative fundamental class $[\bar{M}, \partial \bar{M}]$ gives an invariant associated with $\rho$.

Definition 2.1 (D2). For a representation $\rho: \pi_{1}(M) \rightarrow G$, define the invariant

$$
\operatorname{Vol}_{2}(\rho)=\left\langle\left(c \circ\left(i_{b}^{*}\right)^{-1} \circ \rho_{b}^{*}\right)[\Theta]_{c, b},[\bar{M}, \partial \bar{M}]\right\rangle .
$$

In definition $\mathrm{D} 2$, a specific continuous bounded volume class $[\Theta]_{c, b}$ in $H_{c, b}^{n}(G, \mathbb{R})$ is involved. The question is naturally raised as to whether, if another continuous bounded volume class is used in D2 instead of $[\Theta]_{c, b}$, the value of the volume of representations changes or not. One could expect that definition D2 does not depend on the choice of continuous bounded volume class but it does not seem easy to get an answer directly. It turns out that D2 is independent of the choice of continuous bounded volume class. For a proof, see Section 5.

Proposition 2.2. Definition D2 does not depend on the choice of continuous bounded volume class. That is, for any two continuous bounded volume classes $\omega_{b}, \omega_{b}^{\prime} \in H_{c, b}^{n}(G, \mathbb{R})$,

$$
\left\langle\left(c \circ\left(i_{b}^{*}\right)^{-1} \circ \rho_{b}^{*}\right)\left(\omega_{b}\right),[\bar{M}, \partial \bar{M}]\right\rangle=\left\langle\left(c \circ\left(i_{b}^{*}\right)^{-1} \circ \rho_{b}^{*}\right)\left(\omega_{b}^{\prime}\right),[\bar{M}, \partial \bar{M}]\right\rangle .
$$

Bucher, Burger and Iozzi proved the volume rigidity theorem for hyperbolic lattices as follows.

Theorem 2.3 [Bucher et al. 2013]. Let $n \geq 3$. Let $i: \Gamma \hookrightarrow \operatorname{Isom}^{+}\left(\mathbb{M}^{n}\right)$ be a lattice embedding and let $\rho: \Gamma \rightarrow \operatorname{Isom}^{+}\left(\mathbb{I}^{n}\right)$ be any representation. Then

$$
\left|\operatorname{Vol}_{2}(\rho)\right| \leq\left|\operatorname{Vol}_{2}(i)\right|=\operatorname{Vol}\left(\Gamma \backslash \mathbb{M}^{n}\right),
$$

with equality if and only if $\rho$ is conjugated to i by an isometry.

\section{New definition D4}

In this section we give a new definition of volume of representations. It will turn out that the new definition is useful in proving that all the definitions of volume of representations are equivalent.

End compactification. Let $\widehat{M}$ be the end compactification of $M$ obtained by adding one point for each end of $M$. Let $\widetilde{M}$ denote the universal cover of $M$. Define $\widehat{\widetilde{M}}$ to be the space obtained by adding to $\tilde{M}$ one point for each lift of each end of $M$. The points added to $M$ are called ideal points of $M$ and the points added to $\widetilde{M}$ are called ideal points of $\widetilde{M}$. Denote by $\partial \widehat{M}$ the set of ideal points of $M$ and by $\partial \widehat{\widetilde{M}}$ the set of ideal points of $\widetilde{M}$. Let $p: \widetilde{M} \rightarrow M$ be the universal covering map. The covering map $p: \widetilde{M} \rightarrow M$ extends to a map $\hat{p}: \widehat{\vec{M}} \rightarrow \widehat{M}$ and, moreover, the action of $\pi_{1}(M)$ 
on $\tilde{M}$ by covering transformations induces an action on $\widehat{\widetilde{M}}$. The action on $\widehat{\widetilde{M}}$ is not free because each point of $\partial \widehat{\widetilde{M}}$ is stabilized by some peripheral subgroup of $\pi_{1}(M)$.

Note that $\widehat{M}$ can be obtained by collapsing each connected component of $\partial \bar{M}$ to a point. Similarly, $\widehat{\widetilde{M}}$ can be obtained by collapsing each connected component of $\bar{p}^{-1}(\partial \bar{M})$ to a point where $\bar{p}: \widetilde{M} \rightarrow \bar{M}$ is the universal covering map. We denote the collapsing map by $\pi: \widetilde{\bar{M}} \rightarrow \widehat{\widetilde{M}}$.

One advantage of $\widehat{M}$ is the existence of a fundamental class in singular homology. While the top dimensional singular homology of $M$ vanishes, the top dimensional singular homology of $\widehat{M}$ with coefficients in $\mathbb{Z}$ is isomorphic to $\mathbb{Z}$. Moreover, it can be easily seen that $H_{*}(\widehat{M}, \mathbb{R})$ is isomorphic to $H_{*}(\bar{M}, \partial \bar{M}, \mathbb{R})$ in degree $* \geq 2$. Hence the fundamental class of $\widehat{M}$ is well-defined and we denote it by $[\widehat{M}]$.

The cohomology groups. Let $Y$ be a topological space and suppose that a group $L$ acts continuously on $Y$. Then the cohomology group $H^{*}(Y ; L, \mathbb{R})$ associated with $Y$ and $L$ is defined in the following way. Our main reference for this cohomology is [DuPre 1968].

For $k>0$, define

$$
F_{\text {alt }}^{k}(Y, \mathbb{R})=\left\{f: Y^{k+1} \rightarrow \mathbb{R} \mid f \text { is alternating }\right\} .
$$

Let $F_{\text {alt }}^{k}(Y, \mathbb{R})^{L}$ denote the subspace of $L$-invariant functions, where the action of $L$ on $F_{\text {alt }}^{k}(Y, \mathbb{R})$ is given by

$$
(g \cdot f)\left(y_{0}, \ldots, y_{k}\right)=f\left(g^{-1} y_{0}, \ldots, g^{-1} y_{k}\right),
$$

for $f \in F_{\text {alt }}^{k}(Y), g \in L$. Define a coboundary operator $\delta_{k}: F_{\text {alt }}^{k}(Y, \mathbb{R}) \rightarrow F_{\text {alt }}^{k+1}(Y, \mathbb{R})$ by the usual

$$
\left(\delta_{k} f\right)\left(y_{0}, \ldots, y_{k+1}\right)=\sum_{i=0}^{k+1}(-1)^{i} f\left(y_{0}, \ldots, \hat{y}_{i}, \ldots, y_{k+1}\right) .
$$

The coboundary operator restricts to the complex $F_{\text {alt }}^{*}(Y, \mathbb{R})^{L}$. The cohomology $H^{*}(Y ; L, \mathbb{R})$ is defined as the cohomology of this complex. Define $F_{\text {alt }, b}^{*}(Y, \mathbb{R})$ as the subspace of $F_{\text {alt }}^{*}(Y, \mathbb{R})$ consisting of bounded alternating functions. Clearly the coboundary operator restricts to the complex $F_{\text {alt }, b}^{*}(Y, \mathbb{R})^{L}$ and so it defines a cohomology, denoted by $H_{b}^{*}(Y ; L, \mathbb{R})$. In particular, for a manifold $M$, the cohomology $H^{*}\left(\tilde{M} ; \pi_{1}(M), \mathbb{R}\right)$ is actually isomorphic to the group cohomology $H^{*}\left(\pi_{1}(M), \mathbb{R}\right)$, and $H_{b}^{*}\left(\tilde{M} ; \pi_{1}(M), \mathbb{R}\right)$ is isomorphic to the bounded cohomology $H_{b}^{*}\left(\pi_{1}(M), \mathbb{R}\right)$.

Remark 3.1. Let $L$ and $L^{\prime}$ be groups acting continuously on topological spaces $Y$ and $Y^{\prime}$, respectively. Given a homomorphism $\rho: L \rightarrow L^{\prime}$, any $\rho$-equivariant 
continuous map $P: Y \rightarrow Y^{\prime}$ defines a chain map,

$$
P^{*}: F_{\text {alt }}^{*}\left(Y^{\prime}, \mathbb{R}\right)^{L^{\prime}} \rightarrow F_{\text {alt }}^{*}(Y, \mathbb{R})^{L} .
$$

Thus it gives a morphism in cohomology. Let $Q: Y \rightarrow Y^{\prime}$ be another $\rho$-equivariant map. For each $k>0$, one may define

$$
H_{k}\left(y_{0}, \ldots, y_{k}\right)=\sum_{i=0}^{i}(-1)^{k}\left(P\left(y_{0}\right), \ldots, P\left(y_{i}\right), Q\left(y_{i}\right), \ldots, Q\left(y_{k}\right)\right) .
$$

Then by a straightforward computation,

$$
\left(\partial_{k+1} H_{k}+H_{k-1} \partial_{k}\right)\left(y_{0}, \ldots, y_{k}\right)=\left(P\left(y_{0}\right), \ldots, P\left(y_{k}\right)\right)-\left(Q\left(y_{0}\right), \ldots, Q\left(y_{k}\right)\right) .
$$

It follows from the above identity that, for any cocycle $f \in F_{\text {alt }}^{k}\left(Y^{\prime}, \mathbb{R}\right)^{L^{\prime}}$,

$$
\left(P^{*} f-Q^{*} f\right)\left(y_{0}, \ldots, y_{k}\right)=\delta_{k}\left(f \circ H_{k-1}\right)\left(y_{0}, \ldots, y_{k}\right) .
$$

From this usual process in cohomology theory, one could expect that $P$ and $Q$ induce the same morphism in cohomology. However, since $f \circ H_{k-1}$ may not be alternating, $P$ and $Q$ may not induce the same morphism in cohomology.

Since $\Theta: \mathcal{X}^{n+1} \rightarrow \mathbb{R}$ is a $G$-invariant continuous bounded alternating cocycle, it yields a bounded cohomology class $[\Theta]_{b} \in H_{b}^{n}(\mathcal{X} ; G, \mathbb{R})$. Let $\overline{\mathcal{X}}$ be the compactification of $\mathcal{X}$ obtained by adding the ideal boundary $\partial \mathcal{X}$. Extending the $G$-action on $\mathcal{X}$ to $\overline{\mathcal{X}}$, we can define a cohomology $H^{*}(\overline{\mathcal{X}} ; G, \mathbb{R})$ and bounded cohomology $H_{b}^{*}(\overline{\mathcal{X}} ; G, \mathbb{R})$. In the rank-1 case, since the geodesic simplex is well-defined for any $(n+1)$-tuple of points of $\bar{X}$, the cocycle $\Theta$ can be extended to a $G$-invariant alternating bounded cocycle $\bar{\Theta}: \bar{X}^{n+1} \rightarrow \mathbb{R}$. Hence $\bar{\Theta}$ determines a cohomology class $[\bar{\Theta}] \in H^{n}(\overline{\mathcal{X}} ; G, \mathbb{R})$ and $[\bar{\Theta}]_{b} \in H_{b}^{n}(\overline{\mathcal{X}} ; G, \mathbb{R})$.

Let $\widehat{D}: \widehat{\widetilde{M}} \rightarrow \overline{\mathcal{X}}$ be a $\rho$-equivariant continuous map whose restriction to $\widetilde{M}$ is a $\rho$-equivariant continuous map from $\widetilde{M}$ to $\mathcal{X}$. We will consider only such kinds of equivariant maps throughout the paper. Denote by $D: \widetilde{M} \rightarrow \mathcal{X}$ the restriction of $\widehat{D}$ to $\widetilde{M}$. Then $\widehat{D}$ induces a homomorphism in cohomology,

$$
\widehat{D}^{*}: H^{n}(\overline{\mathcal{X}} ; G, \mathbb{R}) \rightarrow H^{n}\left(\widehat{\widetilde{M}} ; \pi_{1}(M), \mathbb{R}\right) .
$$

Note that the action of $\pi_{1}(M)$ on $\widehat{\widetilde{M}}$ is not free and hence $H^{*}\left(\widehat{\widetilde{M}} ; \pi_{1}(M), \mathbb{R}\right)$ may not be isomorphic to $H^{*}(\widehat{M}, \mathbb{R})$. Let $H_{\text {simp }}^{*}(\widehat{M}, \mathbb{R})$ denote the simplicial cohomology induced from a simplicial structure on $\widehat{M}$. Then there is a natural restriction map $H^{*}\left(\widehat{\widehat{M}} ; \pi_{1}(M), \mathbb{R}\right) \rightarrow H_{\text {simp }}^{*}(\widehat{M}, \mathbb{R}) \cong H^{*}(\widehat{M}, \mathbb{R})$. Thus we regard the cohomology class $\widehat{D}^{*}[\bar{\Theta}]$ as a cohomology class of $H^{n}(\widehat{M}, \mathbb{R})$. Let $[\widehat{M}]$ be the fundamental cycle in $H_{n}(\widehat{M}, \mathbb{R}) \cong \mathbb{R}$. 
Definition 3.2 (D4). Let $D: \widetilde{M} \rightarrow \mathcal{X}$ be a $\rho$-equivariant continuous map which is extended to a $\rho$-equivariant map $\widehat{D}: \widehat{\widetilde{M}} \rightarrow \overline{\mathcal{X}}$. Then we define the invariant

$$
\operatorname{Vol}_{4}(\rho, D)=\left\langle\widehat{D}^{*}[\bar{\Theta}],[\widehat{M}]\right\rangle .
$$

As observed before, $\widehat{D}^{*}[\bar{\Theta}]$ may depend on the choice of $\rho$-equivariant map. However, it turns out that the value $\operatorname{Vol}_{4}(\rho, D)$ is independent of the choice of $\rho$-equivariant continuous map as follows.

Proposition 3.3. Let $\rho: \pi_{1}(M) \rightarrow G$ be a representation. Then

$$
\operatorname{Vol}_{2}(\rho)=\operatorname{Vol}_{4}(\rho, D) \text {. }
$$

Proof. Since the continuous bounded cohomology $H_{c, b}^{*}(G, \mathbb{R})$ can be computed isomorphically from the complex $C_{c, b}^{*}(\mathcal{X}, \mathbb{R})_{\text {alt }}$, there is the natural inclusion $C_{c, b}^{*}(\mathcal{X}, \mathbb{R})_{\text {alt }} \subset F_{\text {alt }, b}^{*}(\mathcal{X}, \mathbb{R})$. Denote the homomorphism in cohomology induced from the inclusion by $i_{G}: H_{c, b}^{k}(G, \mathbb{R}) \rightarrow H_{b}^{k}(\mathcal{X} ; G, \mathbb{R})$. Clearly, $i_{G}\left([\Theta]_{c, b}\right)=[\Theta]_{b}$.

The bounded cohomology $H_{b}^{*}\left(\pi_{1}(M), \mathbb{R}\right)$ is obtained by the cohomology of the complex $C_{b}^{*}(\tilde{M}, \mathbb{R})_{\mathrm{alt}}^{\pi_{1}(M)}$. Since $C_{b}^{*}(\tilde{M}, \mathbb{R})_{\text {alt }}=F_{\text {alt }, b}^{*}(\tilde{M}, \mathbb{R})$, the induced map $i_{M}: H_{b}^{k}\left(\pi_{1}(M), \mathbb{R}\right) \rightarrow H_{b}^{k}\left(\tilde{M} ; \pi_{1}(M), \mathbb{R}\right)$ is the identity map. Let $\widehat{D}: \widehat{\widetilde{M}} \rightarrow \overline{\mathcal{X}}$ be a $\rho$-equivariant map which maps $\widetilde{M}$ to $\mathcal{X}$. Then consider the following commutative diagram, where $\pi: \widetilde{\bar{M}} \rightarrow \widehat{\widetilde{M}}$ is the collapsing map:

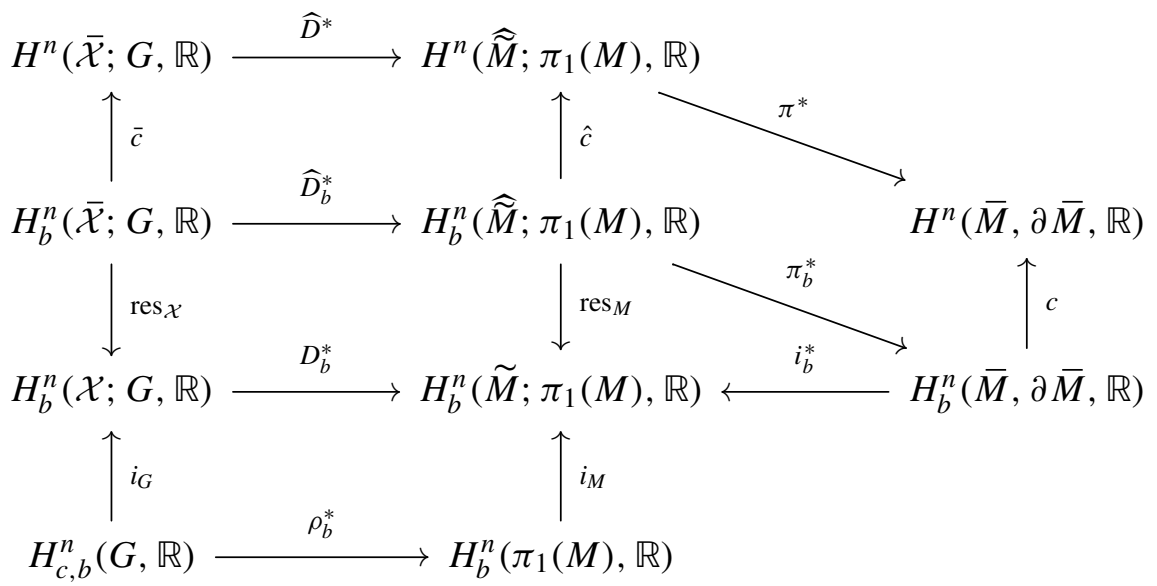

Note that the map $\rho_{b}^{*}$ in the bottom of the diagram is actually induced from the restriction map $D: \widetilde{M} \rightarrow \mathcal{X}$. However, it does not depend on the choice of equivariant map but only on the homomorphism $\rho$. In other words, any continuous equivariant map from $\widetilde{M}$ to $\mathcal{X}$ gives rise to the same map, $\rho_{b}^{*}: H_{c, b}^{*}(G, \mathbb{R}) \rightarrow H_{b}^{*}\left(\pi_{1}(M), \mathbb{R}\right)$. For this reason, we denote it by $\rho_{b}^{*}$ instead of $D_{c, b}^{*}$.

Note that $\pi$ induces a map $\pi^{*}: F_{\text {alt }}^{*}(\widehat{\widetilde{M}}, \mathbb{R}) \rightarrow F_{\text {alt }}^{*}(\widetilde{\bar{M}}, \mathbb{R})$. It follows from the alternating property that the image of $\pi^{*}$ is contained in $C^{*}(\bar{M}, \partial \bar{M}, \mathbb{R})$. Hence the 
map $\pi^{*}: H^{n}\left(\widehat{\widehat{M}} ; \pi_{1}(M), \mathbb{R}\right) \rightarrow H^{n}(\bar{M}, \partial \bar{M}, \mathbb{R})$ makes sense. One can understand $\pi_{b}^{*}: H_{b}^{n}\left(\widehat{\widetilde{M}} ; \pi_{1}(M), \mathbb{R}\right) \rightarrow H_{b}^{n}(\bar{M}, \partial \bar{M}, \mathbb{R})$ in a similar way.

Noting that $\bar{c}\left([\bar{\Theta}]_{b}\right)=[\bar{\Theta}]$ and $\operatorname{res}_{\mathcal{X}}\left([\bar{\Theta}]_{b}\right)=[\Theta]_{b}$, it follows from the above commutative diagram that

$$
\begin{aligned}
\left(\left(i_{b}^{*}\right)^{-1} \circ i_{M} \circ \rho_{b}^{*}\right)[\Theta]_{c, b} & =\left(\left(i_{b}^{*}\right)^{-1} \circ D_{b}^{*} \circ i_{G}\right)[\Theta]_{c, b}=\left(\left(i_{b}^{*}\right)^{-1} \circ D_{b}^{*} \circ \operatorname{res} \mathcal{X}\right)[\bar{\Theta}]_{b} \\
& =\left(\left(i_{b}^{*}\right)^{-1} \circ \operatorname{res}_{M} \circ \widehat{D}_{b}^{*}\right)[\bar{\Theta}]_{b}=\left(\pi_{b}^{*} \circ \widehat{D}_{b}^{*}\right)[\bar{\Theta}]_{b} .
\end{aligned}
$$

Hence

$$
\begin{aligned}
\operatorname{Vol}_{2}(\rho) & =\left\langle\left(c \circ\left(i_{b}^{*}\right)^{-1} \circ i_{M} \circ \rho_{b}^{*}\right)[\Theta]_{c, b},[\bar{M}, \partial \bar{M}]\right\rangle \\
& =\left\langle\left(c \circ \pi_{b}^{*} \circ \widehat{D}_{b}^{*}\right)[\bar{\Theta}]_{b},[\bar{M}, \partial \bar{M}]\right\rangle=\left\langle\left(\pi^{*} \circ \widehat{D}^{*} \circ \bar{c}\right)[\bar{\Theta}]_{b},[\bar{M}, \partial \bar{M}]\right\rangle \\
& =\left\langle\left(\pi^{*} \circ \widehat{D}^{*}\right)[\bar{\Theta}],[\bar{M}, \partial \bar{M}]\right\rangle=\left\langle\widehat{D}^{*}[\bar{\Theta}], \pi_{*}[\bar{M}, \partial \bar{M}]\right\rangle \\
& =\left\langle\widehat{D}^{*}[\bar{\Theta}],[\widehat{M}]\right\rangle=\operatorname{Vol}_{4}(\rho, D) .
\end{aligned}
$$

Proposition 3.3 implies that the value $\operatorname{Vol}_{4}(\rho, D)$ does not depend on the choice of continuous equivariant map. Hence from now on we will use the notation $\operatorname{Vol}_{4}(\rho):=\operatorname{Vol}(\rho, D)$. Furthermore, Proposition 3.3 allows us to interpret the invariant $\operatorname{Vol}_{2}(\rho)$ in terms of a pseudodeveloping map via $\operatorname{Vol}_{4}(\rho)$ in the next section. Note that a pseudodeveloping map for $\rho$ is a specific kind of $\rho$-equivariant continuous map $\widehat{\widetilde{M}} \rightarrow \overline{\mathcal{X}}$.

\section{Pseudodeveloping map and definition D1}

Dunfield [1999] introduced the notion of pseudodeveloping map in order to define the volume of representations $\rho: \pi_{1}(M) \rightarrow \mathrm{SO}(3,1)$ for a noncompact complete hyperbolic 3-manifold $M$ of finite volume. We start by recalling the definition of pseudodeveloping map.

Definition 4.1 (cone map). Let $\mathcal{A}$ be a set, let $t_{0} \in \mathbb{R}$, and let cone $(\mathcal{A})$ be the cone obtained from $\mathcal{A} \times\left[t_{0}, \infty\right]$ by collapsing $\mathcal{A} \times\{\infty\}$ to a point, called $\infty$. A map $\widehat{D}: \operatorname{cone}(\mathcal{A}) \rightarrow \overline{\mathcal{X}}$ is a cone map if $\widehat{D}(\operatorname{cone}(\mathcal{A})) \cap \partial \mathcal{X}=\{\widehat{D}(\infty)\}$ and for all $a \in \mathcal{A}$ the map $\left.\widehat{D}\right|_{a \times\left[t_{0}, \infty\right]}$ is either the constant to $\widehat{D}(\infty)$ or the geodesic ray from $\widehat{D}\left(a, t_{0}\right)$ to $\widehat{D}(\infty)$, parametrized in such a way that the parameter $\left(t-t_{0}\right), t \in\left[t_{0}, \infty\right]$, is the arc length.

For each ideal point $v$ of $M$, fix a product structure $T_{v} \times[0, \infty)$ on the end relative to $v$. The fixed product structure induces a cone structure on a neighborhood of $v$ in $\widehat{M}$, which is obtained from $T_{v} \times[0, \infty]$ by collapsing $T_{v} \times\{\infty\}$ to a point $v$. We lift such structures to the universal cover. Let $\tilde{v}$ be an ideal point of $\tilde{M}$ that projects to the ideal point $v$. Denote by $E_{\tilde{v}}$ the cone at $\tilde{v}$ that is homeomorphic to $P_{\tilde{v}} \times[0, \infty]$, where $P_{\tilde{v}}$ covers $T_{v}$ and $P_{\tilde{v}} \times\{\infty\}$ is collapsed to $\tilde{v}$. 
Definition 4.2 (pseudodeveloping map). Let $\rho: \pi_{1}(M) \rightarrow G$ be a representation. A pseudodeveloping map for $\rho$ is a piecewise-smooth $\rho$-equivariant map $D: \widetilde{M} \rightarrow \mathcal{X}$. Moreover, $D$ is required to extend to a continuous map $\widehat{D}: \widehat{\vec{M}} \rightarrow \overline{\mathcal{X}}$ with the property that there exists a $t \in \mathbb{R}^{+}$such that, for each end $E_{\tilde{v}}=P_{\tilde{v}} \times[0, \infty]$ of $\widehat{\vec{M}}$, the restriction of $\widehat{D}$ to $P_{\tilde{v}} \times[t, \infty]$ is a cone map.

Definition 4.3. A triangulation of $\widehat{M}$ is an identification of $\widehat{M}$ with a complex obtained by gluing together with simplicial attaching maps. It is not required for the complex to be simplicial, but it is required that open simplices embed.

Note that a triangulation of $\widehat{M}$ always exists and it lifts uniquely to a triangulation of $\widehat{\widetilde{M}}$. Given a triangulation of $\widehat{M}$, one can define the straightening of pseudodeveloping maps as follows.

Definition 4.4 (straightening map). Let $\widehat{M}$ be triangulated. Let $\rho: \pi_{1}(M) \rightarrow G$ be a representation and $D: \widetilde{M} \rightarrow \mathcal{X}$ a pseudodeveloping map for $\rho$. A straightening of $D$ is a continuous piecewise-smooth $\rho$-equivariant map $\operatorname{Str}(D): \widehat{\widehat{M}} \rightarrow \overline{\mathcal{X}}$ such that

- for each simplex $\sigma$ of the triangulation, $\operatorname{Str}(D)$ maps $\widetilde{\sigma}$ to $\operatorname{Str}(D \circ \widetilde{\sigma})$,

- for each end $E_{\tilde{v}}=P_{\tilde{v}} \times[0, \infty]$, there exists a $t \in \mathbb{R}$ such that $\operatorname{Str}(D)$ restricted to $P_{\tilde{v}} \times[t, \infty]$ is a cone map,

where $\widetilde{\sigma}$ is a lift of $\sigma$ to $\widehat{\widetilde{M}}$ and $\operatorname{Str}(D \circ \widetilde{\sigma})$ is the geodesic straightening of the map $D \circ \widetilde{\sigma}: \Delta^{n} \rightarrow \overline{\mathcal{X}}$.

Note that any straightening of a pseudodeveloping map is also a pseudodeveloping map.

Lemma 4.5. Let $\widehat{M}$ be triangulated. Let $\rho: \pi_{1}(M) \rightarrow G$ be a representation and $D: \widetilde{M} \rightarrow \mathcal{X}$ a pseudodeveloping map for $\rho$. Then a straightening $\operatorname{Str}(D)$ of $D$ exists and, furthermore, $\operatorname{Str}(D): \widehat{M} \rightarrow \overline{\mathcal{X}}$ is always equivariantly homotopic to $\widehat{D}$ via a homotopy that fixes the vertices of the triangulation.

Proof. First, set $\operatorname{Str}(D)(V)=f(V)$ for every vertex $V$ of the triangulation. Then extend $\operatorname{Str}(D)$ to a map which is piecewise-straight with respect to the triangulation. This is always possible because $\mathcal{X}$ is contractible. Note that $\widehat{D}$ and $\operatorname{Str}(D)$ agree on the ideal vertices of $\widehat{\vec{M}}$ and are equivariantly homotopic via the straight-line homotopy between them. Hence it can be easily seen that the extension is a straightening of $D$.

For any pseudodeveloping map $D: \tilde{M} \rightarrow \mathcal{X}$, for $\rho$,

$$
\int_{M} D^{*} \omega_{\mathcal{X}}
$$

is always finite. This can be seen as follows. We stick to the notation used in Definition 4.2. We may assume that the restriction of $\widehat{D}$ to each $E_{\tilde{v}}=P_{\tilde{v}} \times[0, \infty]$ 
is a cone map. Choose a fundamental domain $F_{0}$ of $T_{v}$ in $P_{\tilde{v}}$. Then there exists a $t \in \mathbb{R}^{+}$such that

$$
\left|\int_{T_{v} \times[t, \infty)} D^{*} \omega_{\mathcal{X}}\right|=\operatorname{Vol}_{n}\left(\operatorname{cone}\left(D\left(F_{0} \times\{t\}\right)\right)\right) \leq \frac{1}{n-1} \operatorname{Vol}_{n-1}\left(D\left(F_{0} \times\{t\}\right)\right),
$$

where $\operatorname{Vol}_{n-1}$ denotes the $(n-1)$-dimensional volume. The last inequality holds for any Hadamard manifold with sectional curvature at most -1 . See [Gromov 1982, Section 1.2]. Hence the integral of $D^{*} \omega_{\mathcal{X}}$ over $M$ is finite.

Definition 4.6 (D1). Let $D: \widetilde{M} \rightarrow \mathcal{X}$ be a pseudodeveloping map for a representation $\rho: \pi_{1}(M) \rightarrow G$. Define the invariant

$$
\operatorname{Vol}_{1}(\rho, D)=\int_{M} D^{*} \omega_{\mathcal{X}}
$$

In the case that $G=\mathrm{SO}(n, 1)$, Francaviglia [2004] showed that definition D1 does not depend on the choice of pseudodeveloping map. We give a self-contained proof for this in the rank-1 case.

Proposition 4.7. Let $\rho: \pi_{1}(M) \rightarrow G$ be a representation. Then, for any pseudodeveloping map $D: \widetilde{M} \rightarrow \mathcal{X}$,

$$
\operatorname{Vol}_{1}(\rho, D)=\operatorname{Vol}_{4}(\rho) .
$$

Thus, $\operatorname{Vol}_{1}(\rho, D)$ does not depend on the choice of pseudodeveloping map.

Proof. Let $\mathcal{T}$ be a triangulation of $\widehat{M}$ with simplices $\sigma_{1}, \ldots, \sigma_{N}$. Then the triangulation gives rise to a fundamental cycle $\sum_{i=1}^{N} \sigma_{i}$ of $\widehat{M}$. Let $\operatorname{Str}(D)$ be a straightening of $D$ with respect to the triangulation $\mathcal{T}$. Since $\operatorname{Str}(D)$ is a $\rho$-equivariant continuous map, we have

$$
\begin{aligned}
\operatorname{Vol}_{4}(\rho):=\operatorname{Vol}_{4}(\rho, D) & =\left\langle\operatorname{Str}(D)^{*}[\bar{\Theta}],[\widehat{M}]\right\rangle=\left\langle\bar{\Theta}, \sum_{i=1}^{N} \operatorname{Str}\left(\widehat{D}\left(\sigma_{i}\right)\right)\right\rangle \\
& =\sum_{i=1}^{N} \int_{\operatorname{Str}\left(\widehat{D}\left(\sigma_{i}\right)\right)} \omega \mathcal{X}=\int_{M} \operatorname{Str}(D)^{*} \omega \mathcal{X} .
\end{aligned}
$$

Since both $\operatorname{Str}(D)$ and $\widehat{D}$ are pseudodeveloping maps for $\rho$ that agree on the ideal points of $\widehat{\widetilde{M}}$, it can be proved, using the same arguments as the proof of [Dunfield 1999, Lemma 2.5.1], that

$$
\int_{M} \operatorname{Str}(D)^{*} \omega_{\mathcal{X}}=\int_{M} D^{*} \omega_{\mathcal{X}}=\operatorname{Vol}_{1}(\rho, D) .
$$

Remark 4.8. While D1 is defined with only a pseudodeveloping map, definition D4 is defined with any equivariant map. This is one advantage of definition D4. By Proposition 4.7, the notation $\operatorname{Vol}_{1}(\rho):=\operatorname{Vol}_{1}(\rho, D)$ makes sense. 


\section{Lipschitz simplicial volume and definition D3}

In this section, $M$ is assumed to be a Riemannian manifold with finite Lipschitz simplicial volume. Gromov [1982, Section 4.4] introduced the Lipschitz simplicial volume of Riemannian manifolds. One can define the Lipschitz constant for each singular simplex in $M$ by giving the Euclidean metrics on the standard simplices. Then the Lipschitz constant of a locally finite chain $c$ of $M$ is defined as the supremum of the Lipschitz constants of all singular simplices occurring in $c$. The Lipschitz simplicial volume of $M$ is defined by the infimum of the $\ell^{1}$-norms of all locally finite fundamental cycles with finite Lipschitz constant. Let $[M]_{\text {Lip }}^{\ell^{1}}$ be the set of all locally finite fundamental cycles of $M$ with finite $\ell^{1}$-seminorm and finite Lipschitz constant. If $[M]_{\text {Lip }}^{\ell^{1}}=\varnothing$, the Lipschitz simplicial volume of $M$ is infinite.

In the case that $[M]_{\mathrm{Lip}}^{\ell^{1}} \neq \varnothing$, we gave a new definition of volume of representations in [Kim and Kim 2014] as follows. A representation $\rho: \pi_{1}(M) \rightarrow G$ induces a canonical pullback map $\rho_{b}^{*}: H_{c, b}^{*}(G, \mathbb{R}) \rightarrow H_{b}^{*}\left(\pi_{1}(M), \mathbb{R}\right) \cong H_{b}^{*}(M, \mathbb{R})$ in continuous bounded cohomology. Hence, for any continuous bounded volume class $\omega_{b} \in H_{c, b}^{n}(G, \mathbb{R})$, we obtain a bounded cohomology class $\rho_{b}^{*}\left(\omega_{b}\right) \in H_{b}^{n}(M, \mathbb{R})$. Then, the bounded cohomology class $\rho_{b}^{*}\left(\omega_{b}\right)$ can be evaluated on $\ell^{1}$-homology classes in $H_{n}^{\ell^{1}}(M, \mathbb{R})$ by the Kronecker products,

$$
\langle\cdot, \cdot\rangle: H_{b}^{*}(M, \mathbb{R}) \otimes H_{*}^{\ell^{1}}(M, \mathbb{R}) \rightarrow \mathbb{R} .
$$

For more details about this, see [Kim and Kim 2014].

Definition 5.1 (D3). We define the invariant

$$
\operatorname{Vol}_{3}(\rho)=\inf \left\langle\rho_{b}^{*}\left(\omega_{b}\right), \alpha\right\rangle,
$$

where the infimum is taken over all $\alpha \in[M]_{\text {Lip }}^{\ell^{1}}$ and all $\omega_{b} \in H_{c, b}^{n}(G, \mathbb{R})$ with $c\left(\omega_{b}\right)=\omega_{\mathcal{X}}$.

One advantage of $\mathrm{D} 3$ is that the isomorphism $H_{b}^{n}(\bar{M}, \partial \bar{M}, \mathbb{R}) \rightarrow H_{b}^{n}(\bar{M}, \mathbb{R})$ is not needed. When $M$ admits the isomorphism above, we will verify that definition D3 is eventually equivalent to the other definitions of volume of representations.

Lemma 5.2. Suppose that $M$ is a noncompact, connected, orientable, aspherical, tame Riemannian manifold with finite Lipschitz simplicial volume and that each end of $M$ has amenable fundamental group. Then, for any $\alpha \in[M]_{\text {Lip }}^{\ell^{1}}$ and any continuous bounded volume class $\omega_{b}$,

$$
\left\langle\rho_{b}^{*}\left(\omega_{b}\right), \alpha\right\rangle=\left\langle\left(c \circ\left(i_{b}^{*}\right)^{-1} \circ \rho_{b}^{*}\right)\left(\omega_{b}\right),[\bar{M}, \partial \bar{M}]\right\rangle .
$$

Proof. When $M$ is a 2-dimensional manifold, the proof is given in [Kim and Kim 2014]. Actually the proof in the general case is the same. We sketch the proof here for the reader's convenience. Let $K$ be a compact core of $M$. Note that $K$ is a 
compact submanifold with boundary that is a deformation retract of $M$. Consider the following commutative diagram, where every map is the map induced from the canonical inclusion:

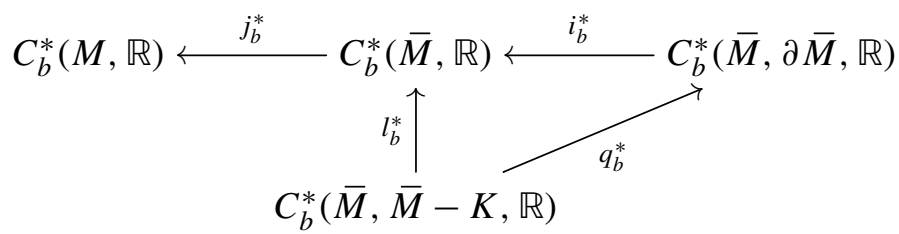

Every map in the diagram induces an isomorphism in bounded cohomology in $* \geq 2$. Thus, there exists a cocycle $z_{b} \in C_{b}^{n}(\bar{M}, \bar{M}-K, \mathbb{R})$ such that $l_{b}^{*}\left(\left[z_{b}\right]\right)=\rho_{b}^{*}\left(\omega_{b}\right)$.

Let $c=\sum_{i=1}^{\infty} a_{i} \sigma_{i}$ be a locally finite fundamental $\ell^{1}$-cycle with finite Lipschitz constant representing $\alpha \in[M]_{\text {Lip }}^{\ell^{1}}$. Then we have

$$
\left\langle\rho_{b}^{*}\left(\omega_{b}\right), \alpha\right\rangle=\left\langle l_{b}^{*}\left(\left[z_{b}\right]\right), \alpha\right\rangle=\left\langle z_{b}, c\right\rangle .
$$

Since $z_{b}$ vanishes on simplices with image contained in $\bar{M}-K$, we have the equality $\left\langle z_{b}, c\right\rangle=\left\langle z_{b},\left.c\right|_{K}\right\rangle$, where $\left.c\right|_{K}=\sum_{\operatorname{im} \sigma_{i} \cap K \neq \varnothing} a_{i} \sigma_{i}$. It is a standard fact that the sum $\left.c\right|_{K}$ represents the relative fundamental class $[\bar{M}, \bar{M}-K]$ in $H_{n}(\bar{M}, \bar{M}-K, \mathbb{R})$ (see [Löh 2007, Theorem 5.3]). On the other hand, we have

$$
\begin{aligned}
\left\langle\left(c \circ\left(i_{b}^{*}\right)^{-1} \circ \rho_{b}^{*}\right)\left(\omega_{b}\right),[\bar{M}, \partial \bar{M}]\right\rangle & \left.=\left\langle\left(c \circ q_{b}^{*}\right)\left(\left[z_{b}\right]\right),[\bar{M}, \partial \bar{M}]\right)\right\rangle \\
& =\left\langle\left[z_{b}\right], q_{*}[\bar{M}, \partial \bar{M}]\right\rangle \\
& =\left\langle\left[z_{b}\right],[\bar{M}, \bar{M}-K]\right\rangle=\left\langle z_{b},\left.c\right|_{K}\right\rangle .
\end{aligned}
$$

By Lemma 5.2 we can reformulate definition D3 as

$$
\operatorname{Vol}_{3}(\rho)=\inf _{\omega_{b}}\left\langle\left(c \circ\left(i_{b}^{*}\right)^{-1} \circ \rho_{b}^{*}\right)\left(\omega_{b}\right),[\bar{M}, \partial \bar{M}]\right\rangle,
$$

where the infimum is taken over all continuous bounded volume classes. Noting that $[\Theta]_{c, b} \in H_{c, b}^{n}(G, \mathbb{R})$ is a continuous bounded volume class, it is clear that

$$
\operatorname{Vol}_{3}(\rho) \leq \operatorname{Vol}_{2}(\rho) .
$$

It is conjecturally true that the comparison map $H_{c, b}^{n}(G, \mathbb{R}) \rightarrow H_{c}^{n}(G, \mathbb{R})$ is an isomorphism for any connected semisimple Lie group $G$ with finite center. Hence, conjecturally, $\operatorname{Vol}_{2}(\rho)=\operatorname{Vol}_{3}(\rho)$. In spite of the absence of a proof of the conjecture, we will give a proof for $\operatorname{Vol}_{2}(\rho)=\operatorname{Vol}_{3}(\rho)$ by using definition D4.

Lemma 5.3. Let $\omega_{b} \in H_{c, b}^{n}(G, \mathbb{R})$ be a continuous bounded volume class, and let $f_{b}: \mathcal{X}^{n+1} \rightarrow \mathbb{R}$ be a continuous bounded alternating $G$-invariant cocycle representing $\omega_{b}$. Then $f_{b}$ is extended to a bounded alternating $G$-invariant cocycle $\bar{f}_{b}: \overline{\mathcal{X}}^{n+1} \rightarrow \mathbb{R}$. Furthermore, $\bar{f}_{b}$ is uniformly continuous on $\mathcal{X}^{n} \times\{\xi\}$ for any $\xi \in \partial \mathcal{X}$. 
Proof. For any $\left(\bar{x}_{0}, \ldots, \bar{x}_{n}\right) \in \overline{\mathcal{X}}^{n+1}$, define

$$
\bar{f}_{b}\left(\bar{x}_{0}, \ldots, \bar{x}_{n}\right)=\lim _{t \rightarrow \infty} f_{b}\left(c_{0}(t), \ldots, c_{n}(t)\right),
$$

where each $c_{i}(t)$ is a geodesic ray toward $\bar{x}_{i}$. Here, for $x \in \mathcal{X}$, we say that $c:[0, \infty) \rightarrow \mathcal{X}$ is a geodesic ray toward $x$ if there exists a $t \in[0, \infty)$ such that the restriction map $\left.c\right|_{[0, t]}$ of $c$ to $[0, t]$ is a geodesic with $c(t)=x$ and $\left.c\right|_{[t, \infty)}$ is constant to $x$. Then it is clear that $\bar{f}_{b}\left(x_{0}, \ldots, x_{n}\right)=f_{b}\left(x_{0}, \ldots, x_{n}\right)$ for $\left(x_{0}, \ldots, x_{n}\right) \in \mathcal{X}^{n+1}$. To see the well-definedness of $\bar{f}_{b}$, we need to show that, for other geodesic rays $c_{i}^{\prime}(t)$ toward $\bar{x}_{i}$,

$$
\lim _{t \rightarrow \infty} f_{b}\left(c_{0}(t), \ldots, c_{n}(t)\right)=\lim _{t \rightarrow \infty} f_{b}\left(c_{0}^{\prime}(t), \ldots, c_{n}^{\prime}(t)\right) .
$$

Note that the limit always exists because $f_{b}$ is bounded. In the rank- 1 case, the distance between two geodesic rays with the same endpoint decays exponentially to 0 as they go to the endpoint. Moreover, since $f_{b}$ is $G$-invariant and $G$ transitively acts on $\mathcal{X}$, we have that $f_{b}$ is uniformly continuous on $\mathcal{X}^{n+1}$. Thus, for any $\epsilon>0$, there exists some number $T>0$ such that

$$
\left|f_{b}\left(c_{0}(t), \ldots, c_{n}(t)\right)-f_{b}\left(c_{0}^{\prime}(t), \ldots, c_{n}^{\prime}(t)\right)\right|<\epsilon
$$

for all $t>T$. This implies (2) and hence $\bar{f}_{b}$ is well-defined.

The alternating property of $\bar{f}_{b}$ actually comes from $f_{b}$. Due to the alternating property of $f_{b}$, we have

$$
\begin{aligned}
\bar{f}_{b}\left(\bar{x}_{0}, \ldots, \bar{x}_{i}, \ldots, \bar{x}_{j}, \ldots, \bar{x}_{n}\right) & =\lim _{t \rightarrow \infty} f_{b}\left(c_{0}(t), \ldots, c_{i}(t), \ldots, c_{j}(t), \ldots, c_{n}(t)\right) \\
& =\lim _{t \rightarrow \infty}-f_{b}\left(c_{0}(t), \ldots, c_{j}(t), \ldots, c_{i}(t), \ldots, c_{n}(t)\right) \\
& =-\bar{f}_{b}\left(\bar{x}_{0}, \ldots, \bar{x}_{j}, \ldots, \bar{x}_{i}, \ldots, \bar{x}_{n}\right) .
\end{aligned}
$$

Therefore, we conclude that $\bar{f}_{b}$ is alternating. The boundedness and $G$-invariance of $\bar{f}_{b}$ immediately follows from the boundedness and $G$-invariance of $f_{b}$. Furthermore, it is easy to check that $\bar{f}_{b}$ is a cocycle by a direct computation.

Now it remains to prove that $\bar{f}_{b}$ is uniformly continuous on $\mathcal{X}^{n} \times\{\xi\}$. It is obvious that $\bar{f}_{b}$ is continuous on $\mathcal{X}^{n} \times\{\xi\}$. Noting that the parabolic subgroup of $G$ stabilizing $\xi$ acts on $\mathcal{X}$ transitively, it can be easily seen that $\bar{f}_{b}$ is uniformly continuous on $\mathcal{X}^{n} \times\{\xi\}$.

The existence of $\bar{f}_{b}$ allows us to reformulate $\mathrm{Vol}_{3}$ in terms of $\mathrm{Vol}_{4}$. Following the proof of Proposition 3.3, we get

$$
\left\langle\left(c \circ\left(i_{b}^{*}\right)^{-1} \circ \rho_{b}^{*}\right)\left(\omega_{b}\right),[\bar{M}, \partial \bar{M}]\right\rangle=\left\langle\widehat{D}^{*}\left[\bar{f}_{b}\right],[\widehat{M}]\right\rangle .
$$

The last term $\left\langle\widehat{D}^{*}\left[\bar{f}_{b}\right],[\widehat{M}]\right\rangle$ above is computed by $\left\langle\widehat{D}^{*} \bar{f}_{b}, \hat{c}\right\rangle$ for any equivariant map $\widehat{D}$ and fundamental cycle $\hat{c}$ of $\widehat{M}$. By choosing the proper equivariant map 
and fundamental cycle, we will show that $\left\langle\widehat{D}^{*}\left[\bar{f}_{b}\right],[\widehat{M}]\right\rangle$ does not depend on the choice of continuous bounded volume class.

Proposition 5.4. Let $\omega_{b}$ and $\omega_{b}^{\prime}$ be continuous bounded volume classes, and let $\bar{f}_{b}$ and $\bar{f}_{b}^{\prime}$ be the bounded alternating cocycles in $F_{\text {alt }}^{n}(\bar{X} ; G, \mathbb{R})$ associated with $\omega_{b}$ and $\omega_{b}^{\prime}$ respectively, as in Lemma 5.3. Then

$$
\left\langle\widehat{D}^{*}\left[\bar{f}_{b}\right],[\widehat{M}]\right\rangle=\left\langle\widehat{D}^{*}\left[\bar{f}_{b}^{\prime}\right],[\widehat{M}]\right\rangle .
$$

Proof. It suffices to prove that, for some $\rho$-equivariant map $\widehat{D}: \widehat{\widetilde{M}} \rightarrow \overline{\mathcal{X}}$ and fundamental cycle $\hat{c}$ of $\widehat{M}$,

$$
\left\langle\widehat{D}^{*} \bar{f}_{b}, \hat{c}\right\rangle=\left\langle\widehat{D}^{*} \bar{f}_{b}^{\prime}, \hat{c}\right\rangle .
$$

To show this, we will prove that, for some sequence $\left(\hat{c}_{k}\right)_{k \in \mathbb{N}}$ of fundamental cycles of $\widehat{M}$,

$$
\lim _{k \rightarrow \infty}\left(\left\langle\widehat{D}^{*} \bar{f}_{b}, \hat{c}_{k}\right\rangle-\left\langle\widehat{D}^{*} \bar{f}_{b}^{\prime}, \hat{c}_{k}\right\rangle\right)=0 .
$$

Let $v_{1}, \ldots, v_{s}$ be the ideal points of $M$. As in Section 4, fix a product structure $T_{v_{i}} \times[0, \infty]$ on the end relative to $v_{i}$ for each $i=1, \ldots, s$ and then lift such structures to the universal cover. We stick to the notation used in Section 4. Set

$$
M_{k}=M-\bigcup_{i=1}^{s} T_{v_{i}} \times(k, \infty] .
$$

Then $\left(M_{k}\right)_{k \in \mathbb{N}}$ is an exhausting sequence of compact cores of $M$. The boundary $\partial M_{k}$ of $M_{k}$ consists of $\bigcup_{i=1}^{s} T_{v_{i}} \times\{k\}$. Let $\mathcal{T}_{0}$ be a triangulation of $M_{0}$. Then we extend it to a triangulation on $\widehat{M}$ as follows. First note that $\mathcal{T}_{0}$ induces a triangulation on each $T_{v_{i}}$. Let $\tau$ be an $(n-1)$-simplex of the induced triangulation on $T_{v_{i}}$ for some $i \in\{1, \ldots, s\}$. Then we attach $\pi(\tau \times[0, \infty])$ to $T_{v_{i}} \times\{0\}$ along $\tau \times\{0\}$, where $\pi: \bar{M} \rightarrow \widehat{M}$ is the collapsing map. Since $\pi$ is an embedding on $\tau \times[0, \infty)$ and $\pi$ maps $\tau \times\{\infty\}$ to the ideal point $v_{i}$, it can be easily seen that cone $(\tau):=\pi(\tau \times[0, \infty])$ is an $n$-simplex. Hence we can obtain a triangulation of $\widehat{M}$ by attaching each cone $(\tau)$ to $\partial M_{0}$, which is denoted by $\widehat{\mathcal{T}_{0}}$.

Next, we extend $\mathcal{T}_{0}$ to a triangulation of $M_{k}$. In fact, $M_{k}$ is decomposed as

$$
M_{k}=M_{0} \cup \bigcup_{i=1}^{s} T_{v_{i}} \times[0, k] .
$$

Hence we can attach each $\tau \times[0, k]$ to $M_{0}$ along $\tau \times\{0\}$ and then triangulate $\tau \times[0, k]$ by using the prism operator [Hatcher 2002, Chapter 2.1]. Via this process, we obtain a triangulation of $M_{k}$, denoted by $\mathcal{T}_{k}$. Note that $\mathcal{T}_{0}$ and $\mathcal{T}_{k}$ induce the same triangulation on each $T_{v_{i}}$. In addition, one can obtain a triangulation $\widehat{\mathcal{T}_{k}}$ of $\widehat{M}$ from $\mathcal{T}_{k}$ similarly to how $\widehat{\mathcal{T}}_{0}$ is obtained from $\mathcal{T}_{0}$ above. 
Let $c_{k}$ be the relative fundamental class of $\left(M_{k}, \partial M_{k}\right)$ induced from $\mathcal{T}_{k}$. Then it can be seen that

$$
\hat{c}_{k}=c_{k}+(-1)^{n+1} \operatorname{cone}\left(\partial c_{k}\right)
$$

is the fundamental cycle of $\widehat{M}$ induced from $\widehat{\mathcal{T}}_{k}$. Any simplex occurring in $c_{k}$ is contained in $M_{k}$. Now we choose a pseudodeveloping map $\widehat{D}: \widehat{\vec{M}} \rightarrow \bar{X}$. Let $\tilde{v}_{i}$ be a lift of $v_{i}$ to $\widehat{\widetilde{M}}$. Let $P_{\tilde{v}_{i}} \times[0, \infty]$ be the cone structure of a neighborhood of $\tilde{v}_{i}$, where $P_{\tilde{v}_{i}}$ covers $T_{v_{i}}$ and $P_{\tilde{v}_{i}} \times\{\infty\}$ is just the ideal point $\tilde{v}_{i}$. We may assume that $\widehat{D}$ is a cone map on each $P_{\tilde{v}_{i}} \times[0, \infty]$. Let $\tilde{c}_{k}$ be a lift of $c_{k}$ to a cochain in $\widetilde{M}$ and let $\widetilde{\partial c}_{k}$ be a lift of $\partial c_{k}$. Let $\tau \times\{0\}$ be an $(n-1)$-simplex in $T_{v_{i}} \times\{0\}$ occurring in $\partial c_{0}$ and let $\tilde{\tau}$ be a lift of $\tau$ to $P_{\tilde{v}_{i}}$. Then $\tilde{\tau} \times\{k\}$ is a lift of $\tau \times\{k\} \in \partial c_{k}$. Since $\widehat{D}$ is a cone map on $P_{\tilde{v}_{i}} \times[0, \infty]$, we have that $D(\tilde{\tau} \times[0, \infty])$ is the geodesic cone over $\tilde{\tau} \times\{0\}$ with top point $\tilde{v}_{i}$ in $\overline{\mathcal{X}}$. Hence the diameter of $D(\tilde{\tau} \times\{k\})$ decays exponentially to 0 as $k \rightarrow \infty$ for each $\tau$.

By a direct computation, we have

$$
\begin{aligned}
\left\langle\widehat{D}^{*} \bar{f}_{b}-\widehat{D}^{*} \bar{f}_{b}^{\prime}, \hat{c}_{k}\right\rangle & =\left\langle\widehat{D}^{*} \bar{f}_{b}-\widehat{D}^{*} \bar{f}_{b}^{\prime}, \tilde{c}_{k}\right\rangle+(-1)^{n+1}\left\langle\widehat{D}^{*} \bar{f}_{b}-\widehat{D}^{*} \bar{f}_{b}^{\prime}, \operatorname{cone}\left(\widetilde{\partial c_{k}}\right)\right\rangle \\
& =\left\langle\bar{f}_{b}-\bar{f}_{b}^{\prime}, \widehat{D}_{*}\left(\tilde{c}_{k}\right)\right\rangle+(-1)^{n+1}\left\langle\bar{f}_{b}-\bar{f}_{b}^{\prime}, \widehat{D}_{*}\left(\operatorname{cone}\left(\widetilde{\partial c_{k}}\right)\right)\right\rangle \\
& =\left\langle f_{b}-f_{b}^{\prime}, D_{*}\left(\tilde{c}_{k}\right)\right\rangle+(-1)^{n+1}\left\langle\bar{f}_{b}-\bar{f}_{b}^{\prime}, \widehat{D}_{*}\left(\operatorname{cone}\left(\tilde{\partial c_{k}}\right)\right)\right\rangle .
\end{aligned}
$$

The last equality comes from the fact that $\widehat{D}_{*}\left(\tilde{c}_{k}\right)$ is a singular chain in $\mathcal{X}$. Since $f_{b}$ and $f_{b}^{\prime}$ are continuous bounded alternating cocycles representing the continuous volume class $\omega_{\mathcal{X}} \in H_{c}^{n}(G, \mathbb{R})$, there is a continuous alternating $G$-invariant function $\beta: \mathcal{X}^{n} \rightarrow \mathbb{R}$ such that $f_{b}-f_{b}^{\prime}=\delta \beta$. Hence

$$
\left\langle f_{b}-f_{b}^{\prime}, D_{*}\left(\tilde{c}_{k}\right)\right\rangle=\left\langle\delta \beta, D_{*}\left(\tilde{c}_{k}\right)\right\rangle=\left\langle\beta, \partial D_{*}\left(\tilde{c}_{k}\right)\right\rangle=\left\langle\beta, D_{*}\left(\widetilde{\partial c_{k}}\right)\right\rangle .
$$

As observed before, since the diameter of all simplices occurring in $D_{*}\left(\widetilde{\partial c_{k}}\right)$ decays to 0 as $k \rightarrow \infty$ and, moreover, $\beta$ is uniformly continuous on $\mathcal{X}$, we have

$$
\lim _{k \rightarrow \infty}\left\langle\beta, D_{*}\left(\widetilde{\partial c_{k}}\right)\right\rangle=0
$$

Note that $D(\operatorname{cone}(\tilde{\tau} \times\{k\}))$ is the geodesic cone over $D(\tilde{\tau} \times\{k\})$ with top point $\tilde{v}_{i}$. By Lemma 5.3, both $\bar{f}_{b}$ and $\bar{f}_{b}^{\prime}$ are uniformly continuous on $\mathcal{X}^{n} \times\left\{\tilde{v}_{i}\right\}$. Since the diameter of $D(\tilde{\tau} \times\{k\})$ decays to 0 as $k \rightarrow \infty$,

$$
\lim _{k \rightarrow \infty}\left\langle\bar{f}_{b}, D(\operatorname{cone}(\tilde{\tau} \times\{k\}))\right\rangle=\lim _{k \rightarrow \infty}\left\langle\bar{f}_{b}^{\prime}, D(\operatorname{cone}(\tilde{\tau} \times\{k\}))\right\rangle=0 .
$$

Applying this to each $\tau$, we can conclude that

$$
\lim _{k \rightarrow \infty}\left\langle\bar{f}_{b}, D_{*}\left(\operatorname{cone}\left(\widetilde{\partial c}_{k}\right)\right)\right\rangle=\lim _{k \rightarrow \infty}\left\langle\bar{f}_{b}^{\prime}, D_{*}\left(\operatorname{cone}\left(\widetilde{\partial c_{k}}\right)\right)\right\rangle=0 .
$$


In the end, it follows that

$$
\lim _{k \rightarrow \infty}\left\langle\widehat{D}^{*} \bar{f}_{b}-\widehat{D}^{*} \bar{f}_{b}^{\prime}, \hat{c}_{k}\right\rangle=0 .
$$

As we mentioned, the value on the left-hand side does not depend on $\hat{c}_{k}$. Thus we can conclude that $\left\langle\widehat{D}^{*} \bar{f}_{b}-\widehat{D}^{*} \bar{f}_{b}^{\prime}, \hat{c}_{k}\right\rangle=0$. This implies that $\left\langle\widehat{D}^{*} \bar{f}_{b}, \hat{c}\right\rangle=\left\langle\widehat{D}^{*} \bar{f}_{b}^{\prime}, \hat{c}\right\rangle$ for any fundamental cycle $\hat{c}$ of $\widehat{M}$, which completes the proof.

Combining Proposition 5.4 with (3), Proposition 2.2 immediately follows.

Proposition 5.5. The definitions of D3 and D4 are equivalent.

Proof. By Lemma 5.2 and Proposition 3.3, we have

$$
\begin{aligned}
\operatorname{Vol}_{3}(\rho) & =\inf \left\{\left\langle\rho_{b}^{*}\left(\omega_{b}\right), \alpha\right\rangle \mid c\left(\omega_{b}\right)=\omega_{\mathcal{X}} \text { and } \alpha \in[M]_{\mathrm{Lip}}^{\ell^{1}}\right\} \\
& =\inf \left\{\left\langle\left(c \circ\left(i_{b}^{*}\right)^{-1} \circ \rho_{b}^{*}\right)\left(\omega_{b}\right),[\bar{M}, \partial \bar{M}]\right\rangle \mid c\left(\omega_{b}\right)=\omega_{\mathcal{X}}\right\} \\
& =\inf \left\{\left\langle\widehat{D}^{*}\left[\bar{f}_{b}\right],[\widehat{M}]\right\rangle \mid c\left(\omega_{b}\right)=\omega_{\mathcal{X}}\right\}=\left\langle\widehat{D}^{*}[\bar{\Theta}],[\widehat{M}]\right\rangle=\operatorname{Vol}_{4}(\rho) .
\end{aligned}
$$

\section{References}

[Besson et al. 2007] G. Besson, G. Courtois, and S. Gallot, "Inégalités de Milnor-Wood géométriques", Comment. Math. Helv. 82:4 (2007), 753-803. MR 2009e:53055 Zbl 1143.53040

[Borel and Wallach 2000] A. Borel and N. Wallach, Continuous cohomology, discrete subgroups, and representations of reductive groups, 2nd ed., Mathematical Surveys and Monographs 67, American Mathematical Society, Providence, RI, 2000. MR 2000j:22015 Zbl 0980.22015

[Bucher et al. 2012] M. Bucher, M. Burger, R. Frigerio, A. Iozzi, C. Pagliantini, and M. B. Pozzetti, "Isometric properties of relative bounded cohomology", preprint, 2012. arXiv 1205.1022

[Bucher et al. 2013] M. Bucher, M. Burger, and A. Iozzi, "A dual interpretation of the GromovThurston proof of Mostow rigidity and volume rigidity for representations of hyperbolic lattices", pp. 47-76 in Trends in harmonic analysis (Rome, 2011), edited by M. A. Picardello, Springer INdAM Ser. 3, Springer, Milan, 2013. MR 3026348 Zbl 1268.53056

[Burger et al. 2010] M. Burger, A. Iozzi, and A. Wienhard, "Surface group representations with maximal Toledo invariant”, Ann. of Math. (2) 172:1 (2010), 517-566. MR 2012j:22014 Zbl 1208.32014

[Dunfield 1999] N. M. Dunfield, "Cyclic surgery, degrees of maps of character curves, and volume rigidity for hyperbolic manifolds", Invent. Math. 136:3 (1999), 623-657. MR 2000d:57022 Zbl 0928.57012

[DuPre 1968] A. M. DuPre, III, "Real Borel cohomology of locally compact groups", Trans. Amer. Math. Soc. 134 (1968), 239-260. MR 38 \#2240 Zbl 0201.55603

[Francaviglia 2004] S. Francaviglia, "Hyperbolic volume of representations of fundamental groups of cusped 3-manifolds", Int. Math. Res. Not. 2004:9 (2004), 425-459. MR 2004m:57032 Zbl 1088. 57015

[Francaviglia and Klaff 2006] S. Francaviglia and B. Klaff, "Maximal volume representations are Fuchsian”, Geom. Dedicata 117 (2006), 111-124. MR 2007d:51019 Zbl 1096.51004

[Goldman 1982] W. M. Goldman, "Characteristic classes and representations of discrete subgroups of Lie groups”, Bull. Amer. Math. Soc. (N.S.) 6:1 (1982), 91-94. MR 83b:22012 Zbl 0493.57011 
[Gromov 1982] M. Gromov, "Volume and bounded cohomology", Inst. Hautes Études Sci. Publ. Math. 56 (1982), 5-99. MR 84h:53053 Zbl 0516.53046

[Guichardet 1980] A. Guichardet, Cohomologie des groupes topologiques et des algèbres de Lie, Textes Mathématiques 2, CEDIC, Paris, 1980. MR 83f:22004 Zbl 0464.22001

[Hatcher 2002] A. Hatcher, Algebraic topology, Cambridge University Press, 2002. MR 2002k:55001 Zbl 1044.55001

[Inoue and Yano 1982] H. Inoue and K. Yano, "The Gromov invariant of negatively curved manifolds", Topology 21:1 (1982), 83-89. MR 82k:53091 Zbl 0469.53038

[Ivanov 1985] N. V. Ivanov, “Основания теории ограниченных когомологий”, Zap. Nauchn. Sem. Leningrad. Otdel. Mat. Inst. Steklov. (LOMI) 143 (1985), 69-109. Translated as "Foundations of the theory of bounded cohomology" in J. Soviet Math. 37:3 (1987), 1090-1115. MR 87b:53070 Zbl 0573.55007

[Kim and Kim 2014] S. Kim and I. Kim, "Volume invariant and maximal representations of discrete subgroups of Lie groups", Math. Z. 276:3-4 (2014), 1189-1213. MR 3175177 Zbl 1292.22003

[Kim and Kuessner 2015] S. Kim and T. Kuessner, "Simplicial volume of compact manifolds with amenable boundary”, J. Topol. Anal. 7:1 (2015), 23-46. MR 3284388 Zbl 1310.53040

[Löh 2007] C. Löh, $\ell^{1}$-homology and simplicial volume, thesis, Westfälische Wilhelms-Universität, Münster, 2007, available at http://nbn-resolving.de/urn:nbn:de:hbz:6-37549578216. Zbl 1152.57304

[Monod 2001] N. Monod, Continuous bounded cohomology of locally compact groups, Lecture Notes in Mathematics 1758, Springer, Berlin, 2001. MR 2002h:46121 Zbl 0967.22006

Received January 6, 2015.

SUNGWOON KIM

DEPARTMENT OF MATHEMATICS

JEJU NATIONAL UNIVERSITY

102 JEJUDAEHAK-RO

JEJU 63243

REPUBLIC OF KOREA

sungwoon@jejunu.ac.kr 


\title{
PACIFIC JOURNAL OF MATHEMATICS
}

\author{
msp.org/pjm
}

Founded in 1951 by E. F. Beckenbach (1906-1982) and F. Wolf (1904-1989)

\section{EDITORS}

Don Blasius (Managing Editor)

Department of Mathematics

University of California

Los Angeles, CA 90095-1555

blasius@math.ucla.edu

\author{
Paul Balmer \\ Department of Mathematics \\ University of California \\ Los Angeles, CA 90095-1555 \\ balmer@math.ucla.edu \\ Robert Finn \\ Department of Mathematics \\ Stanford University \\ Stanford, CA 94305-2125 \\ finn@math.stanford.edu \\ Sorin Popa \\ Department of Mathematics \\ University of California \\ Los Angeles, CA 90095-1555 \\ popa@math.ucla.edu
}

\author{
Vyjayanthi Chari \\ Department of Mathematics \\ University of California \\ Riverside, CA 92521-0135 \\ chari@math.ucr.edu \\ Kefeng Liu \\ Department of Mathematics \\ University of California \\ Los Angeles, CA 90095-1555 \\ liu@math.ucla.edu \\ Jie Qing \\ Department of Mathematics \\ University of California \\ Santa Cruz, CA 95064 \\ qing@ cats.ucsc.edu
}

\section{PRODUCTION}

Silvio Levy, Scientific Editor, production@msp.org

\section{SUPPORTING INSTITUTIONS}

ACADEMIA SINICA, TAIPEI

CALIFORNIA INST. OF TECHNOLOGY

INST. DE MATEMÁTICA PURA E APLICADA

KEIO UNIVERSITY

MATH. SCIENCES RESEARCH INSTITUTE

NEW MEXICO STATE UNIV.

OREGON STATE UNIV.

\author{
STANFORD UNIVERSITY \\ UNIV. OF BRITISH COLUMBIA \\ UNIV. OF CALIFORNIA, BERKELEY \\ UNIV. OF CALIFORNIA, DAVIS \\ UNIV. OF CALIFORNIA, LOS ANGELES \\ UNIV. OF CALIFORNIA, RIVERSIDE \\ UNIV. OF CALIFORNIA, SAN DIEGO \\ UNIV. OF CALIF., SANTA BARBARA
}

\author{
Daryl Cooper \\ Department of Mathematics \\ University of California \\ Santa Barbara, CA 93106-3080 \\ cooper@math.ucsb.edu \\ Jiang-Hua Lu \\ Department of Mathematics \\ The University of Hong Kong \\ Pokfulam Rd., Hong Kong \\ jhlu@maths.hku.hk \\ Paul Yang \\ Department of Mathematics \\ Princeton University \\ Princeton NJ 08544-1000 \\ yang@math.princeton.edu
}

These supporting institutions contribute to the cost of publication of this Journal, but they are not owners or publishers and have no responsibility for its contents or policies.

See inside back cover or msp.org/pjm for submission instructions.

The subscription price for 2016 is US $\$ 440 /$ year for the electronic version, and $\$ 600 /$ year for print and electronic.

Subscriptions, requests for back issues and changes of subscribers address should be sent to Pacific Journal of Mathematics, P.O. Box 4163, Berkeley, CA 94704-0163, U.S.A. The Pacific Journal of Mathematics is indexed by Mathematical Reviews, Zentralblatt MATH, PASCAL CNRS Index, Referativnyi Zhurnal, Current Mathematical Publications and Web of Knowledge (Science Citation Index).

The Pacific Journal of Mathematics (ISSN 0030-8730) at the University of California, c/o Department of Mathematics, 798 Evans Hall \#3840, Berkeley, CA 94720-3840, is published twelve times a year. Periodical rate postage paid at Berkeley, CA 94704, and additional mailing offices. POSTMASTER: send address changes to Pacific Journal of Mathematics, P.O. Box 4163, Berkeley, CA 94704-0163.

PJM peer review and production are managed by EditFLOW ${ }^{\circledR}$ from Mathematical Sciences Publishers.

\section{PUBLISHED BY}

\section{mathematical sciences publishers \\ nonprofit scientific publishing}

http://msp.org/

(C) 2016 Mathematical Sciences Publishers 


\section{PACIFIC JOURNAL OF MATHEMATICS}

Volume $280 \quad$ No. $1 \quad$ January 2016

Stable capillary hypersurfaces in a wedge

JAIGYOUNG CHOE and MIYUKI KoISO

The Chern-Simons invariants for the double of a compression body

DAVID L. DUNCAN

Compactness and the Palais-Smale property for critical Kirchhoff equations in

closed manifolds

EMMANUEL HeBEy

On the equivalence of the definitions of volume of representations

SUNGWOON KIM

Strongly positive representations of even GSpin groups

YEANSU KIM

An Orlik-Raymond type classification of simply connected 6-dimensional torus manifolds with vanishing odd-degree cohomology

\section{SHINTARÔ KUROKI}

Solutions with large number of peaks for the supercritical Hénon equation

Zhongyuan LiU and ShuangJie Peng

Effective divisors on the projective line having small diagonals and small heights and 141 their application to adelic dynamics

YÛSUKE OKUYAMA

Computing higher Frobenius-Schur indicators in fusion categories constructed from inclusions of finite groups

\section{Peter Schauenburg}

Chordal generators and the hydrodynamic normalization for the unit ball

SEBASTIAN SCHLEISSINGER

On a question of A. Balog

ILYA D. SHKREDOV

Uniqueness result on nonnegative solutions of a large class of differential inequalities 241 on Riemannian manifolds

YUHUA SUN

Correction to "Closed orbits of a charge in a weakly exact magnetic field"

WILL J. MERRY 